\title{
Modelling of Ion Transport in Solids with a General Bond Valence Based Force-Field
}

\author{
S. Adams ${ }^{*}$ and R.P. Rao \\ Department of Materials Science and Engineering, \\ National University of Singapore, Singapore 117574, Singapore
}

\section{ARTICLE INFO}

Article history:

Received 11 October 2010

Received in revised form 26 October 2010

Accepted 28 October 2010

Keywords:

Bond valence

Electrode

Lithium ion battery

Lithium ion pathways

Molecular dynamic method

Solid electrolyte

\begin{abstract}
A B S T R A C T
Empirical bond length - bond valence relations provide insight into the link between structure of and ion transport in solid electrolytes. Building on our earlier systematic adjustment of bond valence (BV) parameters to the bond softness, here we discuss how the squared BV mismatch can be linked to the absolute energy scale and used as a general Morse-type interaction potential for analyzing low-energy pathways in ion conducting solid or mixed conductors either by an energy landscape approach or by molecular dynamics (MD) simulations. For a wide range of Lithium oxides we could thus model ion transport revealing significant differences to an earlier geometric approach. Our novel BV-based force-field has also been applied to investigate a range of mixed conductors, focusing on cathode materials for lithium ion battery (LIB) applications to promote a systematic design of LIB cathodes that combine high energy density with high power density. To demonstrate the versatility of the new BV-based force-field it is applied in exploring various strategies to enhance the power performance of safe low cost LIB materials $\left(\mathrm{LiFePO}_{4}, \mathrm{LiVPO}_{4} \mathrm{~F}, \mathrm{LiFeSO}_{4} \mathrm{~F}\right.$, etc. $)$.
\end{abstract}

(C) 2010 Atom Indonesia. All rights reserved

\section{INTRODUCTION}

Understanding ionic motion in disordered solids obviously requires insight into the correlation between ion mobility and the structural and energetic local environment of the mobile ions. Local structure models for disordered solid electrolytes such as ion conducting glasses may in principle be derived from diffraction data (crystal structure refinements for crystalline phases or reverse Monte Carlo (RMC) fitting for glassy phases) or molecular dynamics (MD) simulations $[1,2]$. In principle, MD simulations permit to derive comprehensive structural and dynamical information within the limitations imposed by the system size, the simulated period and the agreement of the employed interaction potential parameters with reality. Effectively both approaches have shown to be valuable tools in obtaining insights into the conduction mechanism and its correlation to the atomic structure, though in the case of MD simulations it has to be verified that the force field chosen for the simulations leads to structure models that are consistent with experimental information [2-4].

\footnotetext{
* Corresponding author.

E-mail address: mseasn@nus.edu.sg ( S. Adams )
}

In this work, we discuss how the bond valence (BV) method can be used to predict characteristics of ionic conductivity from structure models [4-7], and be optimized by linking it to an absolute energy scale for a more straightforward comparison with other atomistic simulation and modelling approaches.

\section{EXPERIMENTAL METHODS}

Empirical relationships between bond length $R$ and bond valence $s_{A-X}=\exp \left[\left(R_{0}-R\right) / b\right]$ are widely used in crystal chemistry to identify plausible equilibrium sites for an atom in a structure as sites where the BV sum of the atom matches its oxidation state (see e.g. the recent review by Brown [5] and references therein). In our earlier work, we introduced a systematic adjustment of BV parameters to the bond softness [6-9], which together with the inclusion of interactions beyond the first coordination shell permits more adequate estimates of non-equilibrium site energies. The inclusion of weak interactions to more distant counterions beyond the first coordination shell is indispensable for modelling ion transport pathways as it avoids artefacts in the BV variation, when an ion moves across the border of its coordination shell. Low BV sum mismatch (and hence low 
energy) pathways for a cation $\mathrm{A}^{+}$can then be modelled as regions in the structure, where the bond valence sum $\mathrm{V}(\mathrm{A})=\Sigma \mathrm{s}_{\mathrm{A}-\mathrm{X}}$ (summing up over all adjacent counterions $\mathrm{X}$ ) approaches the ideal valence $V_{\text {id }}(A)$ (i.e. its oxidation state).

To enhance chemical plausibility "BV mismatch landscapes" $|\Delta \mathrm{V}(\mathrm{A})|$ various additional penalty functions $p_{A-X}$ have been introduced that (i) discriminate against sites, where a matching $\mathrm{V}(\mathrm{A})$ is achieved by strongly asymmetric coordinations (details are described in [3,6]) and (ii) exclude sites close to other (immobile) cation types. The cationcation penalty functions may simply take the form of exclusion radii, but truncated Coulomb repulsions yield a more physical description. Interactions among mobile cations are intentionally left out at this stage. While it is obvious that a higher BV mismatch implies an energetically less favourable state, a direct link of the type

$$
E=D_{0} \cdot|\Delta V|^{g}+E_{\text {asym }}+E_{\text {rep }}=D_{0} \cdot\left|\sum_{X} s_{A-X}-V_{i d}(A)\right|^{g}+E_{\text {asym }}+E_{r e p}
$$

remained to be established [4]. In eq. (1), $E_{\text {asym }}$ represents an energy penalty term due to the asymmetry of the coordination of the considered ion and $E_{\text {rep }}$ the (Coulomb) repulsion between mobile and immobile cations.

\section{RESULTS AND DISCUSSION}

\section{Bond valence based force field}

An empirical approach to assess the coefficients $D_{0}$ and $g$ of eq. (1) as well as a suitable functional form for $E_{\text {asym }}$ may start from comparing the interaction distance dependence of the BV sum mismatch with the distance dependence of interaction energy in empirical interatomic potentials. The variation of an individual bond valence can be integrated in a Morse-type potential

$$
E=D_{0}\left\{\left(\exp \left[\alpha\left(R_{\min }-R\right)\right]-1\right)^{2}-1\right\}=D_{0}\left\{\left(\frac{\exp \left[\frac{R_{0}-R}{b}\right]-S_{\min }}{s_{\min }}\right)^{2}-1\right\}
$$

with the BV parameter $b=1 / \alpha$, implying that the interaction energy $E$ can be approximated as a quadratic function of the deviation of the BVsum from its value $s_{\min }=\exp \left[\left(R_{0}-R_{\min }\right) / b\right]$ for the energy minimum distance $\left(R=R_{\min }\right)$ and hence the parameter $g$ in equation (1) is simply 2. Note that the $\mathrm{BV}$ parameter $R_{0}$ (the distance corresponding to $s=1$ ) generally differs from $R_{\min }$ in (the bond distance for which the interaction potential yields an energy minimum). By introducing the relative bond valence $s_{r e l}=s / s_{\min }$ the Morse potential of eq. 2 can be expressed in a compact notation [4]:

$$
E=D_{0}\left\{\frac{\left(s-s_{\min }\right)^{2}}{s_{\min }^{2}}-1\right\}=D_{0}\left(s_{r e l}^{2}-2 s_{r e l}\right)
$$

The Morse-type interaction potential expressed in eq. (2) is characterised by the three parameters $D_{0}$, $R_{\min }$ and $\alpha$ that have to be linked to the $\mathrm{BV}$ parameters. As mentioned above, $\alpha$ can simply be identified with $1 / b$. A tentative approach to establish a consistent set of $R_{\min }$ (or $s_{\min }$ ) values may be expressed as

$$
R_{\min } \approx R_{0} \times\left[0.9185+0.2285 \cdot\left|\sigma_{A}-\sigma_{X}\right|\right]-b \cdot \ln \left(\frac{V_{i d}}{C N}\right)
$$

where $C N$ refers to the preferred coordination number of the central ion (for examples see Table 1) and the empirically determined term in square brackets accounts for the effect of polarisation $\left(\sigma_{\mathrm{A}}\right.$, $\sigma_{\mathrm{X}}$ refer to the absolute softnesses of the cation and anion, respectively; see e.g., ref. [8] ) as well as the influence of higher coordination shells.

For the Morse-type interaction potential expressed in eq. (2) or (3) the dissociation energy $D_{0}$ can be expected to be $D_{0}=b^{2} k / 2, k$ being the force constant at the distance $R=R_{\text {min }}$. We have thus approximated $D_{0}$ for a wide range of cations as

$$
D_{0}=\frac{k \cdot b^{2}}{2}=c \cdot 14.4 \frac{\mathrm{eV}}{\AA} \cdot \frac{\frac{\left(V_{i d}(A) \cdot V_{i d}(X)\right)^{1 / c}}{\sqrt{n_{A} \cdot n_{X}}}}{R_{\min }} \times \frac{b^{2}}{2}
$$

with $c=1$ if A is an $s$ or $p$ block elements, $c=2$ if $\mathrm{s}$ is a $d$ or $f$ block elements. $n_{A}, n_{X}$ represent the principal quantum numbers of cation $A$ and anion $X$ and $\mathrm{V}_{\mathrm{id}}(\mathrm{A}), \mathrm{V}_{\mathrm{id}}(\mathrm{X})$ their respective nominal charges.

In contrast to the conventional $\mathrm{BV}$ sum mismatch description, such BV interaction potentials of the type described in eqs. (2) or (3) fulfil the formal requirements for an anharmonic diatomic interaction potential, allowing for a molecular dynamics simulation based on BV parameters. Due to the ready availability of BV parameters for a wide range of cation anion pairs this might be an attractive option. In table 1 we list as an example parameters derived by the above formalism from our respective softBV bond valence parameters $[8,9]$ for the interaction of 147 cation types with $\mathrm{O}^{2-}$ in compounds that do not contain other types of anions. With these definitions of the energy of a single bond, the total site energy $E(A)$ of a cation $A$, can then be determined as the sum over BV terms for the interactions with each of the $N$ adjacent anions $X_{\mathrm{i}}$ :

$$
E_{p o t}(A)=D_{0}\left[\sum_{j=1}^{N}\left(\frac{s_{A-\chi_{j}}-s_{\min }}{s_{\min }}\right)^{2}-N\right]
$$


By rewriting eq.(6) it becomes obvious that the total potential energy varies with both the mismatch of the bond valence sum and the asymmetry of the coordination. This allows to quantify the correlation between

1. the bond valence sum rule [10], stating that that the sum of the bond valences around an atom is equal to its atomic valence; and

2. and the equal valence rule [10], which states that the sum of the bond valences around any loop is zero, is that the most symmetric distribution of atomic valence among the bonds is energetically preferable.

If for the sake of simplicity only contributions from the $N_{C}$ counterions of type $X$ in the first coordination shell around the cation $A$ are considered in the derivation, the correlation takes the simple form of eq. (7):

$$
E_{p o t}(A)=D_{0}\left[N_{C} \cdot\left\{\left(\frac{V(A)-V_{\min }(A)}{V_{\min }(A)}\right)^{2}-1\right\}+\sum_{i=1}^{N_{C}}\left(\frac{s_{A-X}-\bar{s}_{A-X}}{s_{\min }}\right)^{2}\right]
$$

where $V_{\min }(A)=N_{C} \times s_{\min }$ in the first term (the BV sum mismatch term), while the second term (the asymmetry term) quantifies the effect of the deviation of individual bond valences from their average value $\overline{\mathrm{s}}_{\mathrm{A}-\mathrm{X}}=\mathrm{V}(\mathrm{A}) / \mathrm{N}_{\mathrm{C}}[4]$.

A major advantage of such an energy-scaled BV mismatch is that it allows straightforward combinations of the BV sum term as an effective "attraction term" with suitably weighted penalty functions for coordination asymmetry and particularly a Coulombic cation-cation repulsion. To model Coulomb repulsion we will throughout this paper use fractional charges $q_{A}, q_{X}$ that are calculated based on the formulas

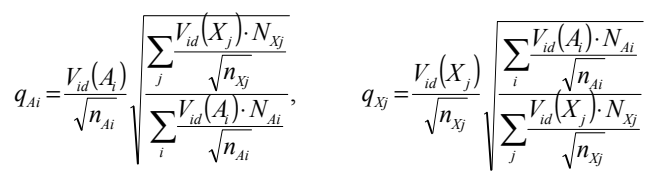

in which $N_{\mathrm{Ai}}, N_{\mathrm{Xj}}$ refer to the occupancies of the i-th cation $A_{i}$ or the j-th anion $X_{j}$ in the structure model (typically 1). This scaling of fractional charges ensures that the model is overall charge-neutral. Obviously, fractional charges from quantum mechanical calculations could be used instead and may improve the quality of the fit, but at the expense of suitability of the approach for the fast and automatic generation of force-fields for screening of a wide range of compounds. The Coulomb repulsions e.g. between immobile $A_{l}$ and mobile $A_{2}$ cations are then taken into account in a screened version $\mathrm{E}_{\text {Coulomb }}\left(A_{1}-A_{2}\right)$ :

$$
E_{\text {Coulomb }}\left(A_{1}-A_{2}\right)=\frac{q_{A 1} \cdot q_{A 2}}{R_{A 1-A 2}} \cdot \operatorname{erfC}\left(\frac{R_{A 1-A 2}}{\rho_{A 1-A 2}}\right)
$$

The screening factor $\boldsymbol{\rho}_{\boldsymbol{A I}-\boldsymbol{A} 2}=\left(\boldsymbol{r}_{A 1}+\boldsymbol{r}_{A 2}\right) \boldsymbol{f}$ therein is assumed to equal the sum of the covalent radii $\boldsymbol{r}_{A i}$ of the two ions involved times a factor $\boldsymbol{f}$ that depends on the average absolute cation electronegativity and the average cation charge in the compound. Typical values of $\boldsymbol{f}$ in ternary and quaternary lithium oxides fall into the range $0.74 \pm 0.04$ and thereby typical values of $\boldsymbol{\rho}$ are of the order of $2 \AA$. While this simplification restricts long range interactions to the real part of the Ewald sum, this localized interaction model has been shown to lead to realistic activation energies of diffusion e.g. for a range of $\mathrm{Li}$ conducting oxyacid salts [11-13]. Due to the favourable convergence of the chosen interaction model a relatively short cut-off radius $(8-10 \AA \AA)$ could be generally used enhancing computational efficiency.

\section{Bond valence Lithium migration maps}

Regions in the structure model, where $E(L i)$ assumes low values, are - as mentioned above assumed to belong to (BV models of) pathways for $\mathrm{Li}^{+}$ion migration. It is assumed that dc conduction requires continuous pathways across the unit cell in at least one dimension. These pathways are visualized as regions enclosed by isosurfaces of constant $E(L i)$ based on calculations of $E(L i)$ for a grid of hypothetical Li positions covering the entire unit cell with a resolution of ca $0.1 \AA$. The threshold value for which isosurfaces of $E(L i)$ form a continuous migration pathway (that includes both occupied and vacant $\mathrm{Li}$ sites), permits a rough estimate of the activation energy for the $\mathrm{Li}^{+}$ion migration. As such an approach neglects relaxation, the assessment of the activation energy is based on an empirical correlation observed for a wide range of Lithium ion conductors. The analysis reveals significant differences to results from a recent geometric Voronoi-Dirichlet partition based study of cages and channels in crystalline Lithium oxides by Anurova et al. [15], which are particularly pronounced for the 33 types of ternary oxides listed in [15] as containing $1 \mathrm{D} \mathrm{Li}$ pathways: In our BV based models 1D migration channels with low to moderate activation energies are observed for 19 of these structures only, while 3 exhibit 2D pathways ( $\mathrm{LT}-\mathrm{LiPO}_{3}, \mathrm{Li}_{2} \mathrm{~W}_{2} \mathrm{O}_{7}, \mathrm{Li}_{2} \mathrm{TeO}_{3}$ ), 6 of them even $3 \mathrm{D}$ pathways $\left(\alpha-\mathrm{Li}_{3} \mathrm{BO}_{3}, \mathrm{Li}_{4} \mathrm{GeO}_{4}, \mathrm{Li}_{2} \mathrm{SeO}_{4}\right.$, $\left.\mathrm{Li}_{2} \mathrm{~T}_{2} \mathrm{O}_{5}(\mathrm{~T}=\mathrm{Si}, \mathrm{Ge}), \mathrm{Li}_{4} \mathrm{TeO}_{5}, \mathrm{Li}_{4} \mathrm{Mo}_{5} \mathrm{O}_{17}\right)$ and the structure models employed in [15] are questionable or implausible or do not yield any paths in further six cases. One of the main reasons for these deviations is the complex curved nature of the higher-dimensional paths, which are difficult to 
identify based on a geometric approach that emphasises straight channels. Unsurprisingly the agreement is much better for the structure types suggested to be 2D- or 3D conductors in ref. [15]. The main difference is however that the pathway analysis yields energy thresholds along the pathways and hence allows a direct assessing of activation energies (Fig. 1).

Table 1. Average cation coordination numbers $\mathrm{N}_{\mathrm{c}}$ in oxides, parameters for bond valence calculations $\left(R_{0}\right.$, cut-off distance $R_{c o}$ ) and the resulting Morse potential parameters $D_{0}, R_{\min }, \alpha$ as determined from a wide range of stable oxide compounds. The BV parameter $b$ is not listed here as it is identical to $1 / \alpha$.

\begin{tabular}{|c|c|c|c|c|c|c|}
\hline cation & $\mathbf{N}_{\mathrm{c}}$ & $\begin{array}{l}\mathbf{R}_{\mathbf{0}} \\
/ \AA \\
\end{array}$ & $\begin{array}{c}\mathbf{R}_{\mathbf{c o}} / \\
\AA \\
\end{array}$ & $\begin{array}{l}\mathbf{D}_{0} \\
/ \mathrm{eV} \\
\end{array}$ & $\begin{array}{r}\mathbf{R}_{\min } \\
/ \AA \AA \\
\end{array}$ & $\begin{array}{r}\alpha=1 / \mathbf{b} \\
/ \AA^{-1} \\
\end{array}$ \\
\hline H(1) & 1.923 & 0.87045 & 4 & 1.8858 & 1.12768 & 2.188184 \\
\hline LI(1) & 5.021 & 1.17096 & 5.5 & 0.98816 & 1.94001 & 1.937984 \\
\hline BE(2) & 4 & 20903 & 5.5 & 2.76882 & 1.52217 & 1.848429 \\
\hline B(3) & 3.417 & .35761 & 4.5 & 2.38924 & 1.34003 & 2.597403 \\
\hline $\mathrm{C}(4)$ & 3 & 1.39826 & 5 & 4.79187 & 1.20089 & 2.237136 \\
\hline $\mathrm{C}(2)$ & 1 & 1.41368 & 5 & 2.40553 & 1.03098 & 2.409639 \\
\hline$N(5)$ & 3 & 1.46267 & 5 & 6.27677 & 1.16142 & 2.222222 \\
\hline$N(3)$ & 2 & 1.40795 & 5 & 3.81089 & 1.13758 & 2.232143 \\
\hline NH4(1) & 3.467 & 2.0338 & 6 & 0.40537 & 2.45364 & 2.262443 \\
\hline NA(1) & 6.52 & 1.56225 & 6 & 0.57523 & 2.37433 & 2.074689 \\
\hline MG(2) & 5.897 & 1.48398 & 5.5 & 1.57554 & 1.95627 & 1.953125 \\
\hline AL(3) & 5.327 & 1.59901 & 5 & 1.80346 & & 2.358491 \\
\hline SI(4) & 4.1 & & 5 & & & 2.314815 \\
\hline $\mathbf{P}(5)$ & 4 & & 5 & & & 2.28833 \\
\hline $\mathbf{P}(\mathbf{3})$ & 3 & 1.51555 & 4.5 & 2.02 & 51 & 2.487562 \\
\hline $\mathbf{S}(6)$ & 4 & 1.6422 & 5 & 4.96726 & 1.38102 & 2.267574 \\
\hline$S(4)$ & 3 & 1.64282 & 5.5 & 3.03672 & 1.41188 & 2.34192 \\
\hline CL(7) & 4 & 1.67946 & 5 & 5.991 & 1.34801 & 2.257336 \\
\hline CL(5) & 3 & 69552 & 5.5 & 4.29089 & 1.35653 & 2.247191 \\
\hline CL(3) & 2 & 72265 & 5.5 & 3.07119 & & 2.03666 \\
\hline K(1) & 8.846 & 1.94117 & 6 & 0.34985 & & 2.293578 \\
\hline CA(2) & 7.544 & 1.79519 & 5.5 & 0.99429 & & 2.10084 \\
\hline $\mathrm{SC}(3$ & 6.255 & & 5.5 & & & 2.024291 \\
\hline TI(4) & 6 & & 5.5 & & & 072 \\
\hline TIC & 6 & & 5.5 & & & 173913 \\
\hline$V(5)$ & 4.166 & & 5.5 & 3 & & .960784 \\
\hline V(4) & 5.738 & 932 & 5 & 47 & & 2.347418 \\
\hline$V(3)$ & 6 & 67799 & 5.5 & 1.82936 & 797 & 2.277904 \\
\hline$V(2$ & 6 & 59976 & 5.5 & 024 & 1.99753 & 2.096436 \\
\hline CR(6) & 4 & & 5.5 & & & 2.10084 \\
\hline CR(5 & 4 & 76781 & 5.5 & 2.36 & & 2.487562 \\
\hline CR(4 & 5.429 & 76095 & 5.5 & 1.93 & 1.762 & 2.444988 \\
\hline CR(3 & 6 & & 5.5 & 1.7 & & 2.325581 \\
\hline C & 5.6 & & 5.5 & & & 2.083333 \\
\hline MN( & 4 & & 6.5 & & & 077 \\
\hline & 4 & & 5.5 & & & 03846 \\
\hline MN( & 4 & & 5.5 & & & 421308 \\
\hline MN( & 5.923 & & 5 & & & 2.487562 \\
\hline MN( & 5.862 & 68993 & 5.5 & 83 & 86 & 2.28833 \\
\hline MN(2) & 5.91 & 62758 & 5.5 & 43 & 2.02969 & 2.079002 \\
\hline FE(3) & & & 5 & & & 2.380952 \\
\hline & & & 5.5 & 1.6926 & & 2.083333 \\
\hline FE(4) & 6 & 1.76559 & 5.5 & 1.87285 & 1.82786 & 2.439024 \\
\hline NI(2) & 5.933 & & 5.5 & & & 2.257336 \\
\hline & 6 & & 5 & & & 2.487562 \\
\hline & 6 & & 5.5 & & & \\
\hline C & 4 & & 5.5 & & & 2.267 \\
\hline $\mathrm{C}$ & 6 & & 5.5 & 24 & 1.7762 & 2.304147 \\
\hline $\operatorname{CO}(2)$ & 5.506 & & 5.5 & 1.51 & 62 & 2.217295 \\
\hline $\operatorname{CO}(1)$ & 2 & .29501 & 5.5 & 2.3 & & 1.610306 \\
\hline & 4 & .70964 & 5 & 242 & 1.70823 & 2.34192 \\
\hline & 2.56 & & 5 & & & 2.227171 \\
\hline CU(1) & 2.56 & 1.5873 & 5 & 0.66417 & 1.78269 & 2.932551 \\
\hline ZN(2) & 4.718 & 1.65344 & 5 & 1.24031 & 1.88557 & 2.48139 \\
\hline GA(3) & 4.905 & 1.71606 & 5 & 1.18456 & 1.79391 & 2.680965 \\
\hline GE(4) & 4.305 & 1.73939 & 5 & 1.91375 & 1.66872 & 2.525253 \\
\hline $\mathbf{A S}(5)$ & 4.029 & & 5 & & & \\
\hline & 3 & & 5 & & & 2.475248 \\
\hline & 4 & & 5.5 & 3.44865 & 1.53287 & 2.403846 \\
\hline & 3 & & 5.5 & 2.38082 & 1.55957 & 2.34192 \\
\hline & 4 & & 5.5 & 4.24339 & 1.50274 & 2.364066 \\
\hline & 10.02 & & 6.5 & & & 2.421308 \\
\hline & 9.4 & 1.95311 & 5.5 & 0.74351 & 2.53589 & 2.197802 \\
\hline$Y(3)$ & 7.285 & 1.90384 & 5.5 & 1.62701 & 2.21523 & 2.09205 \\
\hline
\end{tabular}

\begin{tabular}{|c|c|c|c|c|c|c|}
\hline ZR(4) & 6.765 & 1.84505 & 5.5 & 2.19103 & 1.99602 & $2.04081 \mathrm{C}$ \\
\hline NB(5) & 6.044 & 1.86588 & 5.5 & 2.72326 & 1.85459 & 2.008032 \\
\hline NB(4) & 6 & 1.78543 & 6 & 2.7096 & 1.85989 & 1.901141 \\
\hline NB(3) & 6 & 1.74581 & 6 & 2.02848 & 1.9519 & 1.996008 \\
\hline MO(6) & 4.764 & 1.90934 & 5 & 1.9915 & 1.71254 & 2.557545 \\
\hline MO(5) & 5.98 & 1.8476 & 5.5 & 2.64802 & 1.7867 & 2.07468 \\
\hline MO(4) & 6 & 1.7239 & 6.5 & 3.10807 & 1.85099 & 1.77935 \\
\hline MO(3) & 5.7 & 1.78933 & 5.5 & 1.42826 & 1.92974 & 2.3923 \\
\hline MO(2) & 5.5 & 2.07169 & 5.5 & 0.79706 & 2.3496 & 2.36960 \\
\hline TC(7) & 4 & 1.97036 & 6 & 4.04144 & 1.57518 & 1.94552 \\
\hline RU(6) & 4.5 & 1.92579 & 5.5 & 2.42109 & 1.66431 & 2.3529 \\
\hline RU(5) & 6 & 1.87442 & 5.5 & 2.13208 & 1.81571 & 2.29357 \\
\hline RU(4) & 6 & 1.79363 & 5.5 & 1.99513 & 1.84053 & 2.22717 \\
\hline RU(3) & 6 & 1.72066 & 5.5 & 1.54061 & 1.8932 & 2.32558 \\
\hline RH(4) & 6 & 1.77675 & 5.5 & 1.62725 & 1.81793 & 2.4813 \\
\hline RH(3) & 6 & 1.67013 & 5.5 & 1.92826 & 1.86915 & 2.0920 \\
\hline PD(2) & 4 & 1.62359 & 5.5 & 1.7391 & 1.83671 & 2.00803 \\
\hline PD(4) & 5.333 & 1.805 & 5.5 & 2.04218 & 1.79813 & 2.22717 \\
\hline AG(1) & 4.438 & 1.78239 & 5 & 0.63519 & 2.22578 & $2.5380^{7}$ \\
\hline AG(3) & 6.25 & 1.84687 & 5.5 & 1.52826 & 2.0348 & 2.2522 \\
\hline AG(2) & 5 & 1.72209 & 5.5 & 1.5834 & 2.04171 & 1.9960 \\
\hline $\operatorname{CD}(2)$ & 6.176 & 1.83926 & 5.5 & 0.98346 & 2.1694 & 2.45700 \\
\hline TA(4) & 5.5 & 1.75632 & 6 & & & \\
\hline IN(3) & 6.024 & 1.90305 & 5 & 0.84 & 2.02471 & 2.83286 \\
\hline SN(2) & 3.325 & 1.87499 & 5.5 & 0.97261 & 1.9642 & 2.18340 \\
\hline SN(4) & 6.069 & 1.89019 & 5 & 1.35268 & 1.93422 & 2.63852 \\
\hline SB(5) & 6 & 1.89768 & 5.5 & 1.95523 & 1.86318 & \\
\hline SB(3) & 6 & 1.92036 & 5 & 1.17786 & 2.07526 & 2.36406 \\
\hline I(7) & 5.8 & 1.92274 & 5.5 & 3.21424 & 1.74105 & 2.38663 \\
\hline I(5) & 3.1 & 1.97775 & 6 & 2.48947 & 1.64421 & 2.3584 \\
\hline TE(6) & 6 & 1.91343 & 5.5 & 2.56406 & 1.80876 & 2.42718 \\
\hline TE(4) & 3.396 & 1.9529 & 5.5 & 1.67169 & 1.75208 & $2.4937 t$ \\
\hline TE(2) & 2 & 1.39168 & 6.5 & & & \\
\hline CS(1) & 11.79 & & 6.5 & & & \\
\hline BA(2) & 10.32 & 2.15998 & 6 & 0.579 & 769 & 2.2883 \\
\hline LA(3) & 9.83 & 2.06392 & 5.5 & 1.18 & 2.46989 & 2.21729 \\
\hline LA(3) & 9.83 & 2.06392 & 5.5 & 1.18587 & 2.46989 & 2.21729 \\
\hline CE(4) & 7.867 & 2.02821 & 5.5 & 1.48412 & 2.19872 & 2.25733 \\
\hline CE(3) & 9.147 & 2.03118 & 5.5 & 1.22048 & 2.37861 & 2.22717 \\
\hline PR(3) & 9.067 & 2.03652 & 5.5 & 1.17041 & 2.37113 & $2.2779 c$ \\
\hline ND(3) & 8.647 & 2.02425 & 5.5 & 1.13205 & 2.33016 & 2.3364 \\
\hline SM(3) & 8.119 & 2.01168 & 5.5 & 1.17622 & 2.29536 & 2.30946 \\
\hline EU(3) & 7.743 & 2.00469 & 5.5 & 1.19545 & 2.26888 & 2.3041 \\
\hline EU(2) & 10.111 & 1.89158 & 6 & 32 & 2.53846 & 2.0242 \\
\hline GD(3) & 8.052 & 99654 & 5.5 & .09161 & & 2.40963 \\
\hline TB(4) & 6 & 1.96244 & 6 & 1.70132 & 2.38506 & 2.02429 \\
\hline TB(3) & 7.958 & 1.95675 & 5.5 & 1.20764 & 2.23563 & 2.3094 \\
\hline DY(3) & 7.828 & 1.96029 & 5.5 & 1.1735 & 2.22689 & 2.34741 \\
\hline HO(3) & 7.5 & 1.97099 & 5.5 & 1.12157 & 2.21122 & 2.40963 \\
\hline ER(3) & 7.135 & 1.95608 & 5.5 & 1.12394 & 2.17477 & 2.42718 \\
\hline TM(3) & 6.912 & 1.94901 & 5.5 & 1.18138 & 2.16042 & 2.37529 \\
\hline YB(3) & 6.875 & 1.92872 & 5.5 & 1.21989 & 2.1422 & 2.34741 \\
\hline YB(2) & 8 & 1.63254 & 5.5 & 1.38414 & 2.20942 & 1.9607 \\
\hline $\mathbf{L U}(3)$ & 6.83 & 1.91728 & 5.5 & 1.19488 & 2.136 & 2.3752 \\
\hline HF (4) & 7.105 & & 6 & & 1.99964 & 2.092 \\
\hline TA(5) & 09 & & 5.5 & & 1.85532 & 2.05761 \\
\hline W(6) & 5.688 & 1.90641 & 5 & 1.84267 & 1.77713 & 2.49376 \\
\hline W(5) & 6 & 1.81975 & 6 & 2.6157 & 1.76261 & 2.008032 \\
\hline W(4) & 6 & 1.74558 & 6 & 2.47114 & 1.81945 & 1.923077 \\
\hline RE(7) & 4.098 & 1.97792 & 6 & 3.55593 & 1.59634 & 1.96850 \\
\hline RE(6) & 5.5 & 1.91007 & 6 & 2.95099 & 1.71147 & 2.00803 \\
\hline RE(5) & 6 & 1.82664 & 6 & 2.41099 & 1.76914 & 2.08768 \\
\hline RE(4) & 6 & 1.78237 & 6 & 2.31664 & 1.84497 & 1.97238 \\
\hline RE(3) & 6 & 2.2071 & 6 & 0.81067 & 2.33218 & 2.49376 \\
\hline OS(8) & 5.333 & 1.97728 & 6 & 3.71019 & 1.66146 & 1.95312 \\
\hline OS(7) & 6 & & 5.5 & & 1.72869 & 2.08768 \\
\hline OS(6) & 6 & & 5.5 & & 1.7828 & 2.15982 \\
\hline OS(4) & 6 & & 6 & 2.27524 & 1.81244 & 2.008032 \\
\hline IR(5) & 6 & 1.89791 & 6 & 2.32476 & 1.83476 & 2.087683 \\
\hline $\operatorname{IR}(4)$ & 6 & 1.83233 & 5.5 & 1.68667 & 1.87402 & 2.293578 \\
\hline PT(4) & 6 & 1.82198 & 5.5 & 2.03825 & 1.87174 & 2.087683 \\
\hline PT(3) & 5 & 1.66559 & 5.5 & 2.35953 & 1.81938 & 1.831502 \\
\hline PT(2) & 4 & 1.51205 & 5.5 & 2.14999 & 1.80179 & 1.7421 \\
\hline $\mathbf{A U}(\mathbf{3})$ & 4 & 1.81761 & 5.5 & 1.96967 & 1.81312 & 2.008032 \\
\hline $\mathbf{A U}(\mathbf{1})$ & 2 & 1.71819 & 5.5 & 0.85304 & 1.89543 & 2.26757 \\
\hline HG(2) & 6.966 & 1.81276 & 5.5 & 1.12852 & 2.25275 & 2.15053 \\
\hline HG(1) & 4.786 & 1.8128 & 5.5 & 0.73931 & 2.43155 & 2.15053 \\
\hline TL(3) & 5.22 & 2.06297 & 5 & 0.67637 & 2.10642 & 2.9585 \\
\hline TL(1) & 8.03 & 1.91752 & 6 & 0.34999 & 2.77086 & 2.070393 \\
\hline PB(4) & 5.74 & 2.03293 & 5 & 1.02719 & 2.02857 & 2.824859 \\
\hline PB(2) & 7.541 & 2.01825 & 5.5 & 0.63833 & 2.44191 & 2.309469 \\
\hline BI(5) & 6 & & 5 & & 1.98599 & 2.695418 \\
\hline BI(3) & 6.058 & 2.03677 & 5.5 & 0.97904 & 2.18321 & $2.41545 \mathrm{C}$ \\
\hline
\end{tabular}




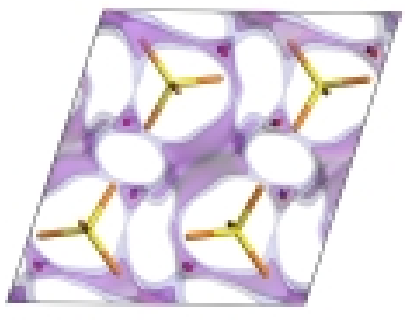

$\mathrm{Li}_{2} \mathrm{SO}_{4}\left(\mathrm{P} 2_{1} / \mathrm{a}, 000058\right)$
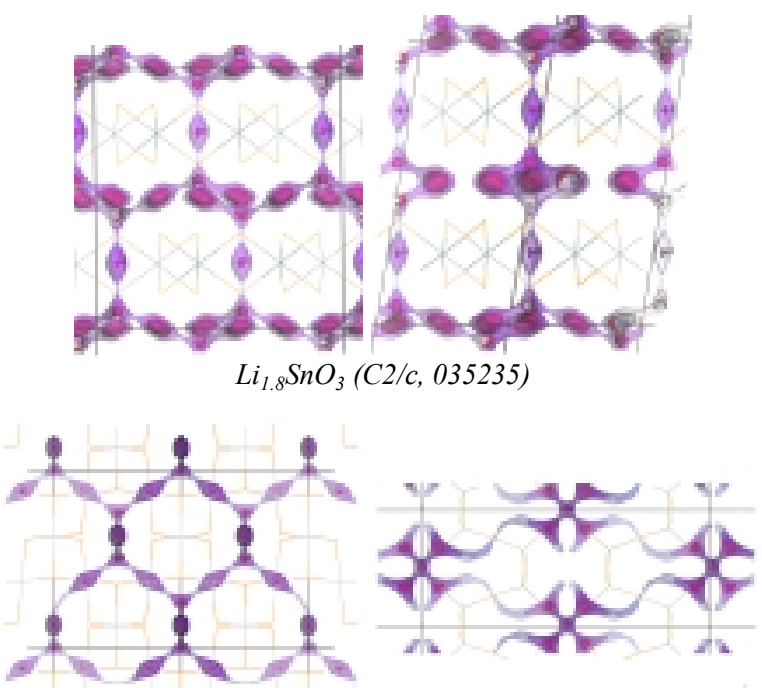

$\mathrm{LiTi}_{2} \mathrm{O}_{4}(\mathrm{Fd}-3 m, 015789)$.

$\mathrm{Li}_{2} \mathrm{Ge}_{2} \mathrm{O} 5(\mathrm{Cc}, 028178)$
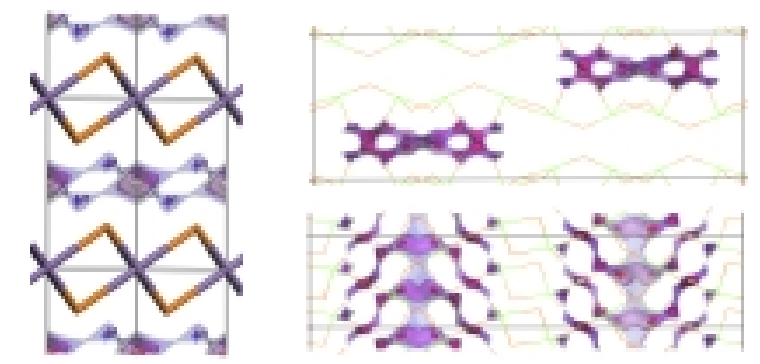

$\mathrm{Li}_{2} \mathrm{MnO}_{2}(\mathrm{P}-3 \mathrm{ml}, 037327)$

$\mathrm{Li}_{2} \mathrm{Te}_{2} \mathrm{O}_{5}$ (Pnaa, 026452)
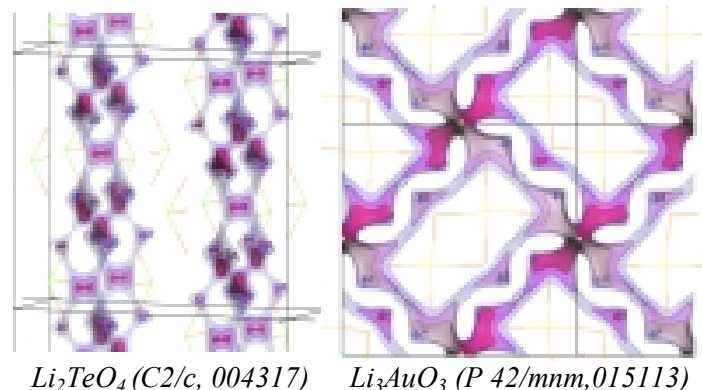

$\mathrm{Li}_{2} \mathrm{TeO}_{4}(\mathrm{C} 2 / \mathrm{c}, 004317)$

$\mathrm{Li}_{3} \mathrm{AuO}_{3}(\mathrm{P}$ 42/mnm,015113)
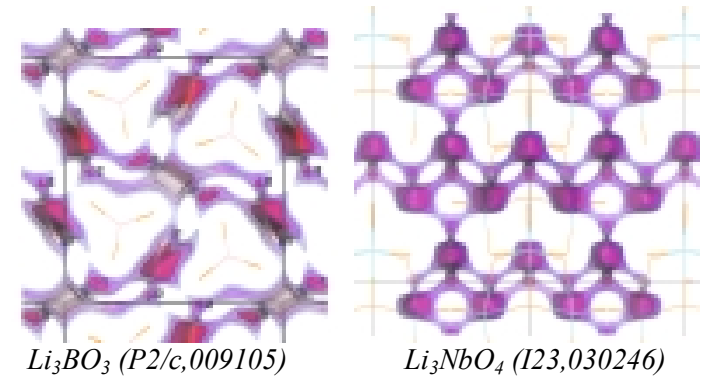
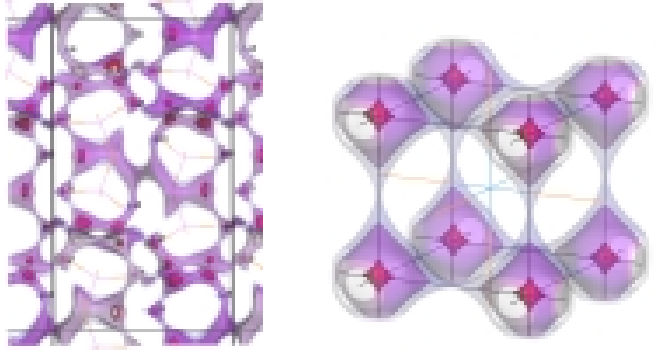

$\mathrm{Li}_{4} \mathrm{P}_{2} \mathrm{O}_{7}\left(\mathrm{P} 2_{1} / n, 039814\right)$

$\mathrm{Li}_{0.93} \mathrm{WO}_{3}(\mathrm{Pm}-3 \mathrm{~m}, 028882)$

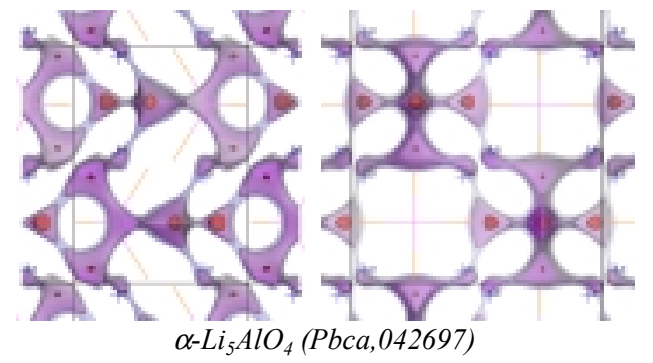

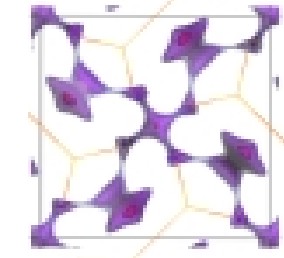

$\mathrm{Li}_{6} \mathrm{Si}_{2} \mathrm{O}_{7}\left(\mathrm{P}-42_{1} m, 025752\right)$

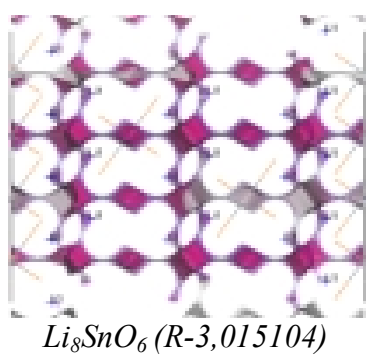

Fig. 1. Examples of isosurfaces of constant $E(L i)$ as bond valence models of $\mathrm{Li}^{+}$ion migration pathways in ternary oxides. In each graph three isosurfaces corresponding to increasing site energies are superimposed (red, magenta, light blue). Li atoms are indicated as blue crosses and labelled. Other atom positions are indicated by line or stick models. Labels below each graph indicate the respective compound name, space group and ICSD database [14] code of the underlying structure data.

\section{Pathways in high energy Lithium-ion battery cathode materials $\mathrm{LiFeSO}_{4} \mathrm{~F}$ and $\mathrm{LiVPO}_{4} \mathrm{~F}$}

Framework materials based on phosphate and sulfate polyanion building blocks are increasingly regarded as favorable replacements for conventional oxide-based cathode materials in lithium-ion battery applications. The lithium insertion phases $\mathrm{LiFePO}_{4}$ and $\mathrm{Li}_{3} \mathrm{~V}_{2}\left(\mathrm{PO}_{4}\right)_{3}$ were the first of such materials identified and characterized. Recently, a few groups have described the insertion properties of the lithium vanadium fluorophosphates, $\mathrm{LiVPO}_{4} \mathrm{~F}$ and $\mathrm{Li}_{5} \mathrm{~V}\left(\mathrm{PO}_{4}\right)_{2} \mathrm{~F}_{2}$ [16-18]. $\mathrm{LiVPO}_{4} \mathrm{~F}$ is iso-structural with the naturally occurring triclinic minerals tavorite $\left(\mathrm{LiFePO}_{4} \mathrm{OH}\right)$ and amblygonite $\left(\mathrm{LiAlPO}_{4} \mathrm{~F}\right)$. The $\mathrm{LiVPO}_{4} \mathrm{~F}$ structure comprises a 3-D framework built up from single chains of 2 distinct cornersharing $\left[\mathrm{VO}_{4} \mathrm{~F}_{2}\right]$ octahedra cross-linked via $\left[\mathrm{PO}_{4}\right]$ 
tetrahedra [16] wherein the oxygen atoms are shared between the two environments. The strong inductive effect of the $\mathrm{PO}_{4}^{3-}$ anion, combined with the presence of structural $\mathrm{F}^{-}$, moderates the energetic of the transition-metal redox couple such that the reversible lithium insertion reactions for $\mathrm{Li}_{1-\mathrm{x}} \mathrm{VPO}_{4} \mathrm{~F}$ occurs at the unusually high operating potential of ca. $4.2 \mathrm{~V}$ versus Li. More recently, analogous fluorosulfates $\mathrm{LiMSO}_{4} \mathrm{~F}(\mathrm{M}=\mathrm{Fe}, \mathrm{Co}, \mathrm{Ni})$ have also been synthesized [19-21] and found to be isostructural to $\mathrm{LiMgSO}_{4} \mathrm{~F}$ and hence to $\mathrm{LiVPO}_{4} \mathrm{~F}$ (though a different setting of the unit cell is chosen in the original publication). As $\mathrm{LiMSO}_{4} \mathrm{~F}(\mathrm{M}=\mathrm{Fe}, \mathrm{Co}, \mathrm{Ni}$ etc.), is unstable at the temperatures of most solid state reactions $\left(\mathrm{LiFeSO}_{4} \mathrm{~F}\right.$ decomposes for temperatures $\geq 350^{\circ} \mathrm{C}$ ) [19], it became accessible only via ionothermal synthesis in EMI-TFSI. Cells with $\mathrm{LiFeSO}_{4} \mathrm{~F}$ cathodes and $\mathrm{Li}$ anodes deliver $85 \%$ of the theoretical specific capacity of $151 \mathrm{mAh} \mathrm{g}^{-1}$ at $\mathrm{C} 1$ rate without carbon coating or nanostructuring. ac conductivity of $\mathrm{LiFeSO}_{4} \mathrm{~F}$ was found to be mainly ionic with $E_{A}=0.99 \pm 0.01 \mathrm{eV}$ and $\sigma(300 \mathrm{~K})$ $\approx 10^{-10} \mathrm{~S} \mathrm{~cm}^{-1}$. From projections of the framework structure. Recham et al. [19] originally suggested 3 tunnels with large cross sections (along [100], [010], and [101]) as pathways for a presumed $3 \mathrm{D}^{+}$ migration.

Here we investigate the characteristics of $\mathrm{Li}^{+}$ ion migration pathways in $\mathrm{LiVPO}_{4} \mathrm{~F}$ and $\mathrm{LiFeSO}_{4} \mathrm{~F}$ using the bond valence (BV) approach and molecular dynamics simulations. Based on a snapshot of the relaxed MD simulated supercell at $300 \mathrm{~K}$ an average crystal structure model is constructed by projecting the positions of all atoms back into a single unit cell. The resulting structure model (Fig. 2-3) closely resembles the published preliminary XRD data. Besides a minor rotation of $\mathrm{SO}_{4}{ }^{2-}$ the main difference is in the $\mathrm{Li}^{+}$distribution, which is characterized by a pronounced disorder along channels extending in the [111] direction with two weak Li density maxima. The averaging of $\mathrm{Li}$ positions around the occupancy maxima yields the two $\mathrm{Li}$ positions: $\mathrm{Li}(1)$ at $0.145,0.5710 .633$ with an occupancy $n=0.6$; and $\operatorname{Li}(2)$ at $0.450,0.854$, 0.854 with $n=0.4$. The two partially occupied sites form $\operatorname{Li}(2)-\operatorname{Li}(1)-\operatorname{Li}(1)-\operatorname{Li}(2)$ channels \| [111] with distances ranging from 1.92-2.12 A favorable for $\mathrm{Li}^{+}$transport, while migration in any other direction - especially along the earlier proposed tunnels-requires hop distances $\geq 3.69 \AA$ (Fig. 3).

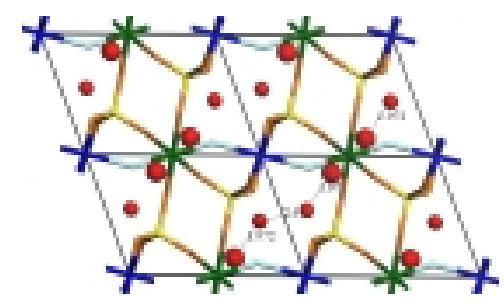

Fig. 2. Model of $\mathrm{LiFeSO}_{4} \mathrm{~F}$ from $\mathrm{MD}$ simulations projected on ac plane ( $\mathrm{Fe}(1)$ green, $\mathrm{Fe}(2)$ blue; $\mathrm{S}$ yellow; $\mathrm{F}$ light blue, $\mathrm{O}$ orange, Li red (occupancy 0.6 for $\operatorname{Li}(1)=$ large spheres, 0.4 for $\operatorname{Li}(2)=$ small spheres) .

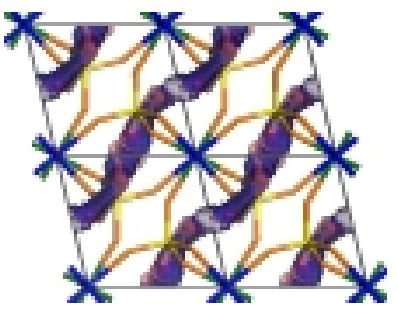

Fig. 3. Comparison of isosurface of constant Li density (dark) from MD trajectory $(\mathrm{T}=600 \mathrm{~K})$ and $\mathrm{BV}$ pathway model (light) in a projection of the $\mathrm{LiFeSO}_{4} \mathrm{~F}$ structure on the $a b$ plane.

Static BV models for the Rietveld-refined and our MD simulated structure models [13] accordingly suggest zig-zag shaped 1D paths $\|[111]$ involving both Li sites as pathways of lowest migration energy barrier $(\approx 0.22 \mathrm{eV})$, while a migration energy of ca. $0.97 \mathrm{eV}$ would be required to connect the channels in the [010] direction and an only slightly higher activation energy of $1.1 \mathrm{eV}$ leads to a 3D network of $\mathrm{Li}^{+}$migration pathways. Since low energy $\mathrm{Li}^{+}$ pathways || [111] connect partially occupied Li sites, a defect formation should not be required for migration along these channels. The experimental $E_{A}=0.99 \mathrm{eV}$ is much higher than the one predicted for migration along [111] channels, but closely resembles the $E_{A}$ for the formation of a 2D pathway network (paths along [111] and [010]).

MD simulations of $\mathrm{LiVPO}_{4} \mathrm{~F}$ have been conducted with GULP [22] using the Morse-type force field and a preliminary version of our $\operatorname{soft} B V$ parameter set. Te starting unit cell was derived from crystallographic data for $\mathrm{LiAlPO}_{4} \mathrm{~F}$ [23]. Simulations were based on a $3 a \times 3 b \times 3 c$ supercell of the conventional setting. Note that the [111] diection in $\mathrm{LiFeSO}_{4} \mathrm{~F}$ corresponds to the c-diection in LiVPO $_{4} \mathrm{~F}$. Geometry was optimized at $\mathrm{T}=300 \mathrm{~K}$. NVT MD simulations were performed at $300 \mathrm{~K}$ to $1000 \mathrm{~K}$ in intervals of $100 \mathrm{~K}$. At each temperature structures are equilibrated, followed by $2 \mathrm{~ns}$ production runs in 2 fs time steps. The diffusion co-efficients obtained from the slopes of the mean square displacement-vs.-time curves of simulations for $\mathrm{T} \geq 600 \mathrm{~K}$ for individual directions indicate that 
the mobility of $\mathrm{Li}+$ is somewhat higher along the z-axis, while the activation is nearly the same (ca.0.5 eV) along all axes (Fig. 4).

$\mathrm{BV}$ models of the $\mathrm{Li}+$ ion migration pathways in $\mathrm{LiVPO}_{4} \mathrm{~F}$ suggest that the continuous pathway of lowest activation energy corresponds to a zig-zag shaped 1-D pathways along the z-axis (Fig. 5a-d).

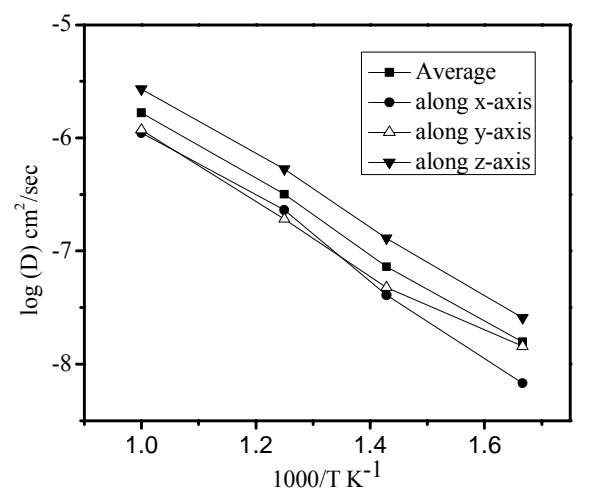

Fig. 4. Arrhenius plot of simulated diffusion coefficient for $\mathrm{Li}^{+}$ion along different Cartesian axes of the model system (with $z \|$ to the c axis).

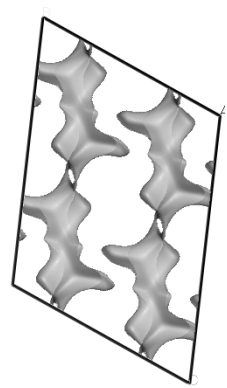

(a)
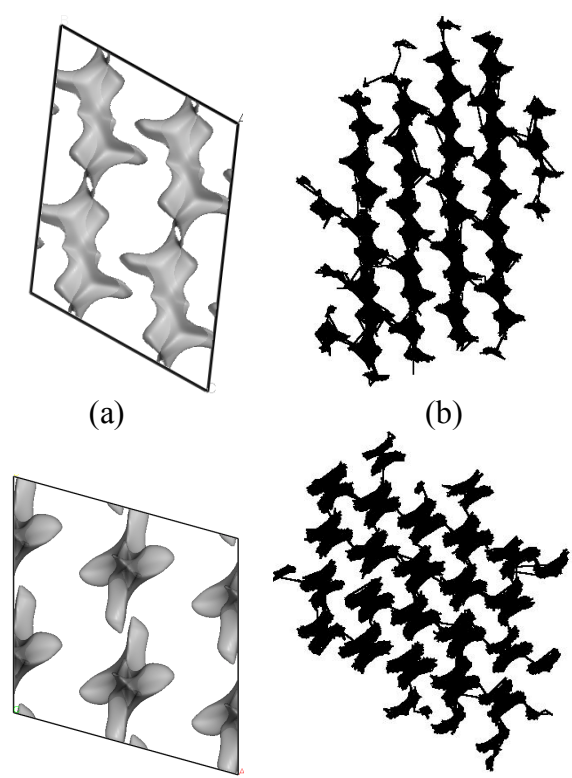

(c) (b)

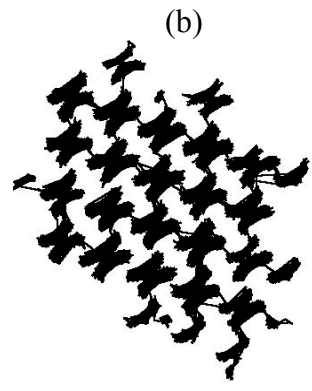

(d)
Fig. 5. BV models of $\mathrm{Li}^{+}$ion migration pathways in $\mathrm{LiVPO}_{4} \mathrm{~F}$ displayed as isosurfaces of constant $\mathrm{Li}$ site energy $E(L i)$ projected (a) on the xz-plane, (c) on the xy plane; compared to traces of $\mathrm{Li}$ motion of $\mathrm{MD}$ simulations in $\mathrm{LiVPO}_{4} \mathrm{~F}$ supercells in matching orientations $(\mathrm{b}, \mathrm{d})$.

\section{MD simulations of $\mathrm{LiFePO}_{4}$ and surface modified $\mathrm{LiFePO}_{4}$ with the $\mathrm{BV}$-based force-field}

MD simulations have been conducted with GULP [22] using the Morse-type force field discussed above for models of bulk $\mathrm{Li}_{0.99} \mathrm{FePO}_{4}$ (with a single built-in $\mathrm{Li}_{\mathrm{Fe}}^{\prime} / \mathrm{Fe}_{\mathrm{Li}}^{\cdot}$ antisite defect per 96 formula units), glassy $\left[\left(\mathrm{Li}_{2} \mathrm{O}\right)_{2 / 3}\left(\mathrm{P}_{2} \mathrm{O}_{5}\right)_{1 / 3}\right]_{411}$ and for a 4367 atoms 3D-periodic interface structure $\left[\mathrm{LiFePO}_{4}\right]_{320^{-}}\left[\left(\mathrm{Li}_{2} \mathrm{O}\right)_{2 / 3}\left(\mathrm{P}_{2} \mathrm{O}_{5}\right)_{1 / 3}\right]_{491}$ for $300 \mathrm{~K} \leq$ $\mathrm{T} \quad \mathrm{v}<1000$ K. Bulk and $\mathrm{LiFePO}_{4}: \mathrm{Li}_{4} \mathrm{P}_{2} \mathrm{O}_{7}$ heterostructure ensembles are equilibrated in NPT simulations over $600 \mathrm{ps}$. The relaxed volume is then fixed and equilibration continued for 150ps at each temperature followed by 600 ps (for $\mathrm{T}=600-1000 \mathrm{~K}$ ), $1 \mathrm{~ns}$ (for $\mathrm{T}=500 \mathrm{~K}$ ), or $3 \mathrm{~ns}$ (for $\mathrm{T}=400-300 \mathrm{~K}$ ) production runs in 1.5 fs steps $[11,13]$. When constructing the interface model based on relaxed structure models of $\mathrm{LiFePO}_{4}$ and glassy $\mathrm{Li}_{4} \mathrm{P}_{2} \mathrm{O}_{7}$, the (010) surface of $\mathrm{LiFePO}_{4} \perp$ to the [010] $\mathrm{Li}^{+}$ migration channels is chosen, since it is both the most relevant surface for $\mathrm{Li}^{+}$transport and the most prominent face of $\mathrm{LiFePO}_{4}$ nanoparticles. The extra charge in non-stoichiometric $\mathrm{Li}_{0.99} \mathrm{FePO}_{4}$ is distributed over all $\mathrm{Fe}$ cations to emulate a charge transfer on a time scale faster than that of ionic transport. Lattice constants and average linear thermal expansion coefficients (for $\mathrm{LiFePO}_{4}$ simulated: $1.8 \times 10^{-5} \mathrm{~K}^{-1}$; experimental: $1.9 \times 10^{-5} \mathrm{~K}^{-1}$, Fig. 6) match literature experiments closely. Simulations for bulk $\mathrm{Li}_{\mathrm{x}} \mathrm{FePO}_{4}(\mathrm{x}=95 / 96)$ yield diffusion coefficients, $D(L i) \| \mathrm{b}$, (Fig. 8) that are consistent with values of $D(L i) \| \mathrm{b}$ derived from the experimental conductivity data by Amin et al. [24], but considerably higher than values reported by $\mathrm{Li}$ et al. [25]. The simulated $E_{A}=0.57 \mathrm{eV}$ for bulk $\mathrm{Li}_{.99} \mathrm{FePO} 4$ is consistent with findings of various experimental and theoretical studies. A BV pathway analysis for both time-averaged and snapshot-type structure models of fully ordered $\mathrm{LiFePO}_{4}$ harmonizes with ab initio studies in yielding zig-zag shaped one-dimensional $\mathrm{Li}^{+}$pathways $\| b$. The apparent contradiction to some of the experimental conductivity data implying a 2D nature of the $\mathrm{Li}^{+}$ motion motivated our study to which extent local structure models that include likely defect scenarios affect the expected pathway dimensionality.

Due to the moderate energetic disadvantage of antisite defects $(\approx 1.1 \mathrm{eV})$ [26] some antisite defects will even occur in equilibrated samples, but concentrations in real samples will be significantly higher due to the common sample non-equilibrium preparation routes and the vicinity of surfaces or interfaces. Antisite defects in $\mathrm{LiFePO}_{4}$ have recently been visualized by scanning transmission electron microscopy [27]. As seen from BV pathway models (Fig. 7 a-d) As to be seen from the BV pathway models in Fig. 1 a, b for a model containing a single antisite defect pair, this defect affects the local $\mathrm{Li}^{+}$ pathways is two ways: $\mathrm{Fe}_{\mathrm{Li}}$ inside the pathway channel blocks the ion transport $\| b$, while $\mathrm{Li}_{\mathrm{Fe}}^{\prime}$ opens up a new path $\perp b$ between the Li channels. 
This can also be seen in MD simulations for elevated temperatures (Fig. 7c,d). For sufficiently high antisite defect concentrations this may lead to long-range $\mathrm{Li}^{+}$transport perpendicular to the channels. Our Monte Carlo-type simulations suggest that the defect concentration required for a $2 \mathrm{D}$ percolating $\mathrm{Li}^{+}$pathway cluster can be reduced to about $2.2 \%$ if a significant energetic preference for the formation of antisite defect pairs close to existing defects is assumed in line with the experimental TEM findings.

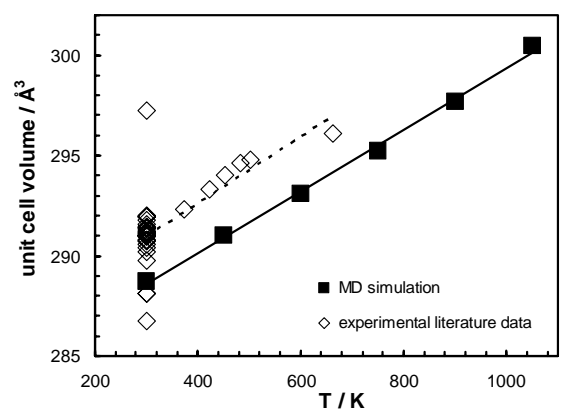

Fig. 6. Temperature dependent lattice constants of $\mathrm{LiFePO}_{4}$ from MD simulations and exp. literature data. (a)

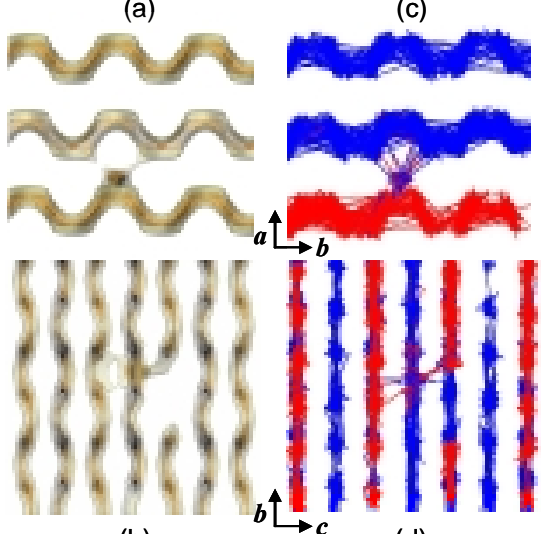

(b) (d)
Fig. 7. $(\mathrm{a}, \mathrm{b})$ Projections of $\mathrm{Li}^{+}$pathway models in $\mathrm{Li}_{99} \mathrm{FePO}_{4}$ with one antisite defect. The 3 super-imposed isosurfaces represent constant $\mathrm{Li}^{+}$site energy characteristic of equilibrium sites (black), migration channels \| b (brown) and migration via antisite defects (beige). The BV based energy landscape approach yields the low-energy pathways even though at this temperature no transport $\perp \mathrm{b}$ is observed during the simulated period of $1.5 \mathrm{~ns}$. For comparison graphs $(\mathrm{c}, \mathrm{d})$ display $\mathrm{Li}^{+}$ trajectories from MD simulations for $T=1000 \mathrm{~K}(1.5 \mathrm{~ns})$. Atoms initially located in $1 \mathrm{~b}$-c layer are marked in red.

Extraordinarily high (dis)charging rates were recently observed for cathodes with the nominal composition $\mathrm{LiFe}_{0.9} \mathrm{P}_{0.95} \mathrm{O}_{4-\delta}$ [28]. Electron microscopy showed that the ca. $50 \mathrm{~nm}$ thin nanocrystals are phase segregated into $\mathrm{Li}_{\mathrm{x}} \mathrm{FePO}_{4}$ and a surface layer of glassy $\mathrm{Li}_{4} \mathrm{P}_{2} \mathrm{O}_{7}$ (or a similar composition) [28-30]. The phase segregation might also involve iron phosphides and/or $\mathrm{Fe}^{3+}$ in the glass, which would raise the electronic conductivity. Our MD simulations show that $D(L i)_{\| b}$ in the $\mathrm{LiFePO}_{4}$ layer is enhanced by ca. 3 orders of magnitude for typical working temperatures (Fig. 8). $D(L i)_{\| b}$ in delithiated heterostructures is similar to the value for $x \approx 1$, while the anisotropy is less pronounced. Due to the reduced activation energy $(=$ migration energy $=0.31 \mathrm{eV}$ for $300 \mathrm{~K} \leq T$ $\leq 700 \mathrm{~K})$, the conductivity enhancement practically vanishes for $T>700 \mathrm{~K}$, where $\mathrm{E}_{\mathrm{A}}$ approaches the bulk value. $\mathrm{Li}^{+}$ions are significantly enriched on the $\mathrm{Li}_{\mathrm{x}} \mathrm{FePO}_{4}$-side of the interface and depleted on the $\mathrm{Li}_{4} \mathrm{P}_{2} \mathrm{O}_{7}$ glass side (Fig. 9). The overall $\mathrm{Li}^{+}$ concentration in the $\mathrm{Li}_{x} \mathrm{FePO}_{4}$ layer increases by $4-7 \%$ (" $x=1$ ") or $17-37 \%$ (" $x=0.06 ")$ significant violation of local electroneutrality within each phase.
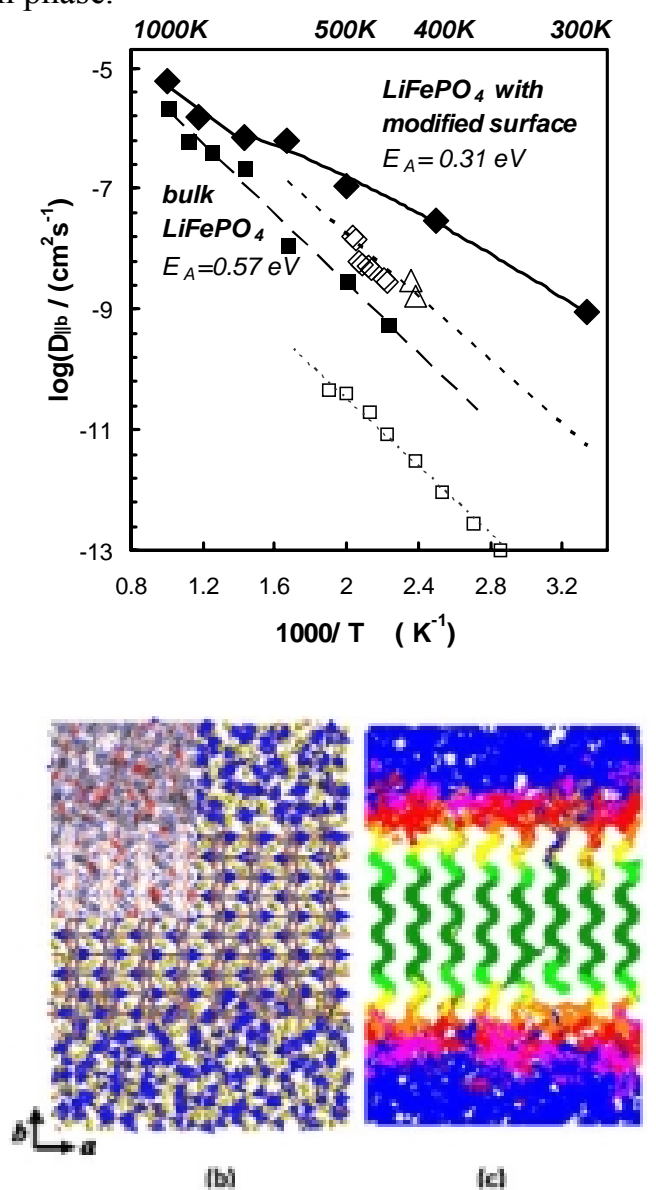

Fig. 8. (a) Comparison of simulation results for the $\mathrm{Li}^{+}$diffusion constant along the channel direction in $\mathrm{LiFePO}_{4}$ from this work ( $\mathbf{m}$ : bulk $\mathrm{LiFePO}_{4}$; : surface-modified $\mathrm{LiFePO}_{4}$ ) with literature data (open symbols, experimental data: $\diamond, \Delta[6], \square[24]$ ). (b) Snapshot of MD simulations of $\mathrm{LiFePO}_{4}: \mathrm{Li}_{4} \mathrm{P}_{2} \mathrm{O}_{7}$ glass heterolayer for $T=700 \mathrm{~K}$ ( $\mathrm{Li}$ : yellow, $\mathrm{PO}_{4}$ : blue tetrahedra, $\mathrm{Fe}$ grey). Inset: superposition of the Li BV pathways in $1 / 8$ of this structure snapshot. Vacant parts of the pathway are marked in red, while pathway regions close to $\mathrm{a}^{+}$are marked in blue. (c) Trace of $\mathrm{Li}^{+}$motion in $\mathrm{LiFePO}_{4}: \mathrm{Li}_{4} \mathrm{P}_{2} \mathrm{O}_{7}$ heterolayer for 500 snapshots over $600 \mathrm{ps}(T=700 \mathrm{~K})$. 

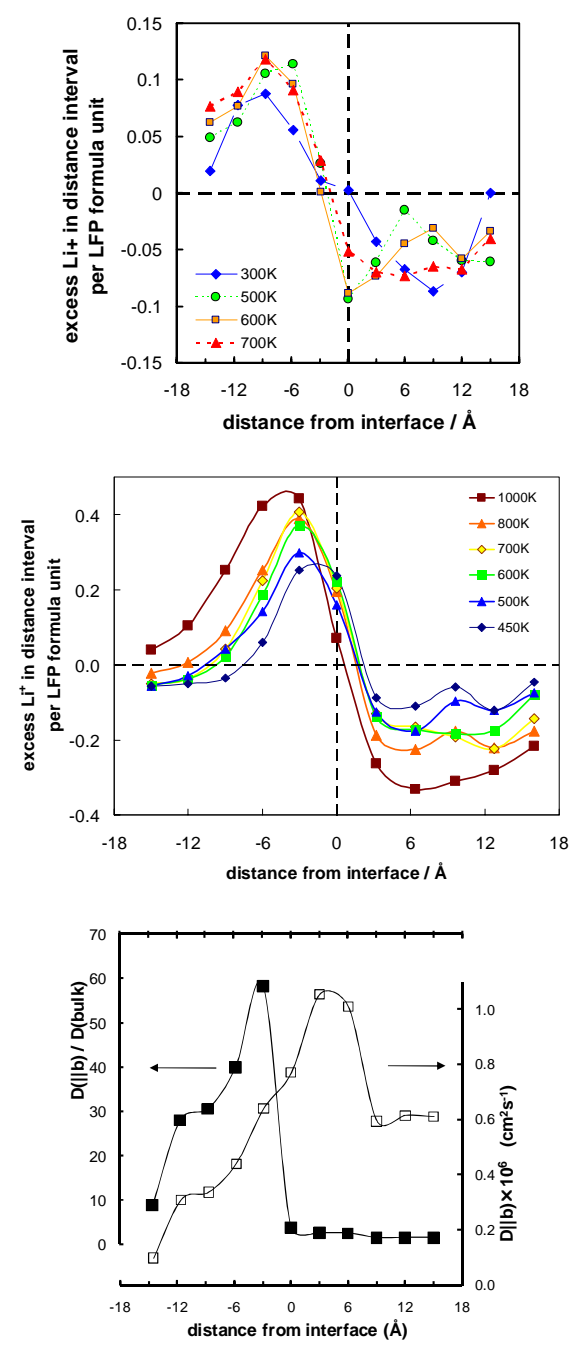

Fig. 9. $\mathrm{Li}^{+}$redistribution at the $\mathrm{Li}_{x} \mathrm{FePO}_{4}: \mathrm{Li}_{4} \mathrm{P}_{2} \mathrm{O}_{7}$ interface displayed as $\%$ excess $\mathrm{Li}^{+}$in ca. $3 \AA$ thick slices vs. distance of the slice from the interface (1.h.s.: $x=1$; centre: $x=0.06$ ). $\mathrm{Li}^{+}$ ions are enriched in the $\mathrm{Li}_{\mathrm{x}} \mathrm{FePO}_{4}$ phase (negative distances), depleted in the $\mathrm{Li}_{4} \mathrm{P}_{2} \mathrm{O}_{7}$ glass. Each value represents an average over 100 - $250 \mathrm{MD}$ time steps. R.h.s.: Variation of ( $\mathbf{m})$ relative $\mathrm{Li}^{+}$diffusion coefficient enhancement (compared to respective bulk values) and ( $\square$ ) absolute $\mathrm{Li}^{+}$diffusion coefficient $\| b$ with the distance of the slice from the interface $(T=600 \mathrm{~K}, \mathrm{x}=1)$.

The change in the extent of $\mathrm{Li}^{+}$redistribution with $x$ implies a fast pseudo-capacitive energy storage component. Moreover, antisite formation near the interface boosts $\mathrm{Li}^{+}$mobility $\perp b$ and promotes a full utilization of the (dis)charging capacity (but slows down transport $\| b$ ). A layer-bylayer analysis of $\mathrm{Li}^{+}$displacements reveals that $\mathrm{Li}^{+}$ mobility $\| b$ is enhanced in the interface region (compared to bulk values) by a factor of $60(\mathrm{~T}=600 \mathrm{~K})$ to ca. $3000(\mathrm{~T}=300 \mathrm{~K})$ in harmony with the maximum of the volume fraction of low BV mismatch regions at the interface (Fig. 6). The relative enhancement is highest in $\mathrm{Li}_{x} \mathrm{FePO}_{4}$, while for $\mathrm{T} \geq 600 \mathrm{~K} D(L i)_{\| b}$ is higher on the glass side of the interface. The anisotropy $D(L i)_{\| b} / D(L i)_{\perp b}$ is gradually reduced from practically infinite in $\mathrm{LiFePO}_{4}$ to (trivially) one within the glass (partly via antisite defects, partly via $\mathrm{Li}^{+}$crossing the interface). Over ca. $1 \mathrm{~nm}$ from the interface $D(L i)_{\perp b}$ in $\mathrm{LiFePO}_{4}$ drops by a factor $\approx 90$. As for " $\mathrm{x}=0.06$ " most $\mathrm{Li}^{+}$reside close to the interface, the overall anisotropy of $D(L i)$ becomes a function of $\mathrm{x}$. Despite their increased concentration in subsurface $\mathrm{LiFePO}_{4}, \mathrm{Li}^{+}$ions find a sufficient number of accessible unoccupied target sites for migration. This is demonstrated in Fig. 10 by the variation of the volume fraction accessible for $\mathrm{Li}^{+}$migration.

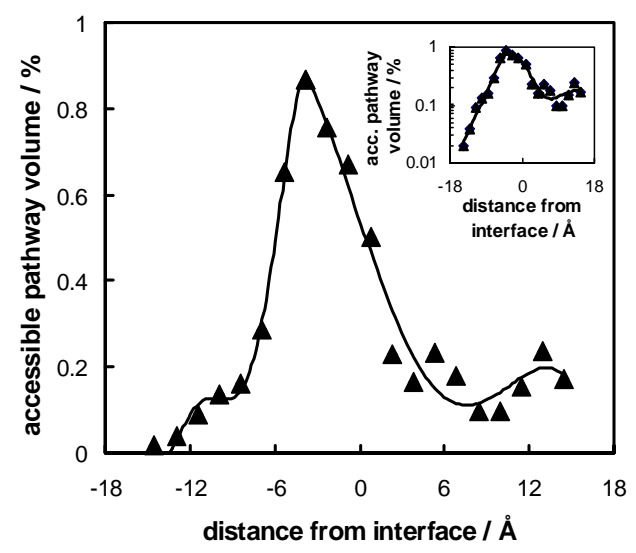

Fig. 10. Variation of accessible pathway volume fraction (regions of low $\mathrm{Li}^{+}$site energy $<0.6 \mathrm{eV}$ that are not blocked by nearby $\mathrm{Li}^{+}$; distance to $\mathrm{Li}>2.5 \AA$ ) within $3 \AA$ slices of MD-simulated $\mathrm{LiFePO}_{4}: \mathrm{Li}_{4} \mathrm{P}_{2} \mathrm{O}_{7}$ vs. distance from interface (averaged over $5 \mathrm{MD}$ time frames at $600 \mathrm{ps}$ intervals, $T=300 \mathrm{~K}$ ). Solid lines: polynomial fits. The logarithmic plot of the same data (inset) highlights the exponential increase of the accessible pathway volume fraction within $\mathrm{LiFePO}_{4}$ when approaching the interface.

\section{CONCLUSIONS}

The bond valence approach has been reworked into an effective local force-field that can be used both to analyse ion transport pathways and fo Molecular dynamics simulations. $\mathrm{LiFeSO}_{4} \mathrm{~F}$ and $\mathrm{LiVPO}_{4} \mathrm{~F}$ are thereby found to be quasi-one dimensional $\mathrm{Li}^{+}$ionic conductors (along channels that for $\mathrm{LiFeSO}_{4} \mathrm{~F}$ extend along [111]). The experimental activation energy and power performance is however controlled by the moderate activation energy for transport perpendicular to the low energy pathways, which (as typical for 1D pathway channels) will in most cases be blocked by defects. The blocking could be overcome by nanostructuring.

The approach can also help to understand the effect of homogeneous and heterogeneous defects on the ionic of $\mathrm{LiFePO}_{4}$, highlighting that the 
dimensionality of ionic motion will depend on the concentration and spatial distribution of antisite defects. Surface modification of $\mathrm{Li}_{x} \mathrm{FePO}_{4}$ nanocrystals by glassy $\mathrm{Li}_{4} \mathrm{P}_{2} \mathrm{O}_{7}$ causes a significant $\mathrm{Li}^{+}$redistribution entailing an enhancement of $\mathrm{Li}^{+}$ mobility and an thus an enhancement of room temperature $\mathrm{Li}^{+}$ion diffusion by about 3 orders of magnitude along the channels. This and the drastically enhanced mobility perpendicular to the $\mathrm{Li}$ channel direction will facilitate a fast (dis)charging of $\mathrm{Li}$ batteries based on surfacemodified $\mathrm{LiFePO}_{4}$ electrodes. The pronounced change in the extent of the $\mathrm{Li}^{+}$redistribution from the phosphate glass layer into subsurface $\mathrm{Li}_{\mathrm{x}} \mathrm{FePO}_{4}$ with $\mathrm{x}$ constitutes an ultrafast pseudocapacitive storage component.

\section{ACKNOWLEDGMENT}

Financial support in the frame of the Singapore Ministry of Education Grant MOE2009T2-1-065 is gratefully acknowledged.

\section{REFERENCES}

1. S. Adams, J. Power Sources 159 (2006) 200.

2. J. Swenson and S. Adams, Phys. Rev. B 63 (2000) 054201.

3. C. Müller E. Zienicke, S. Adams, J. Habasaki and P. Maass, Phys. Rev. B 75 (2007) 014203.

4. S. Adams and R. Prasada Rao, Phys. Chem. 11 (2009) 3210.

5. I.D. Brown, Chem. Rev. 109 (2009) 6858.

6. S. Adams and E. S. Tan, Solid State Ionics $\mathbf{1 7 9}$ (2008) 33.

7. S. Adams, Solid State Ionics 177 (2006) 1625.

8. S. Adams, Acta Crystallogr. B 57 (2001) 278.

9. S. Adams, SoftBV ver. 0.96, http:// www.softBV.net. (2004).

10. I.D. Brown, Acta Crystallogr. B 48 (1992) 553.

11. S. Adams, Journal of Solid State Electrochemistry 14 (2010) 1787.

12. R. Prasada Rao, T.D Tho and S. Adams, Solid State Ionics 181 (2010) 1.

13. S. Adams and R. Prasada Rao, Submitted to Solid State Ionics (2010).
14. ICSD Inorganic Crystal Structure Database, FIZ Karlsruhe.

15. N.A. Anurova, V.A. Blatov, G.D. Ilyushin, O.A. Blatova, A.K. Ivanov-Schitz and L.N. Dem'yanets, Solid State Ionics 179 (2008) 2248.

16. J. Barker, R.K. B. Gover, P. Burns, A. Bryan, M.Y. Saidi and J.L. Swoyer, J. Electrochem. Soc. 152 (2005) A1776.

17. J. Barker, M.Y. Saidi, R.K.B. Gover, P. Burns and A. Bryan, J. Power Sources 174 (2007) 927.

18. S.C. Yin, P. Subramanya Herle, A. Higgins, N.T. Taylor, Y. Makimura and L.F. Nazar, Chem. Mater. 18 (7) (2006) 1745.

19. N. Recham, J.N. Chotard, L. Dupont, C. Delacourt, W. Walker, M. Armand and J.M. Tarascon, Nat. Mater. 9 (2010) 68.

20. J.M. Tarascon, N. Recham, M. Armand, J.N. Chotard, P. Prabanda, W. Walker and L. DuPont, Chem. Mater. 22 (2010) 724.

21. P. Barpanda, N. Recham, J.N. Chotard, K. Djellab, W. Walker, M. Armand and J.M. Tarascon, J. Mater. Chem. 20 (2010) 1659.

22. J.D. Gale, J. Chem. Soc. Faraday Trans. 93 (1997) 629.

23. V.I. Simonov and N.V. Belov, Kristallografiya 3 (1958) 428.

24. R. Amin and J. Maier, Solid State Ionics $\mathbf{1 7 8}$ (2008) 1831; R. Amin, J. Maier, P. Balaya, D.P. Chen and C.T. Lin, Solid State Ionics 179 (2008) 1683.

25. J. Li, W. Yao, S. Martin and D. Vaknin, Solid State Ionics 179 (2008) 2016.

26. C.A.J. Fisher, V.M.H. Prieto and MS. Islam, Chem. Mater. 20 (2008) 5907.

27. S.Y. Chung, S.Y. Choi, T. Yamamoto and Y. Ikuhara, Phys. Rev. Lett. 100 (2008) 125502.

28. B. Kang and G. Ceder, Nature 458 (2009) 190.

29. G. Ceder and B. Kang, J. Power Sources 194 (2009) 1024.

30. K. Zaghib, J.B. Goodenough, A. Mauger and C. Julien, J. Power Sources 194 (2009) 1021. 